\title{
GLOBALE KONZEPTE GEGEN BEDROHUNGEN DURCH AUFKOMMENDE INFEKTIONSKRANKHEITEN
}

Joachim Kreysler

Eine der größten Herausforderungen für die globale Volksgesundheit am Ende des zwanzigsten Jahrhunderts wird heute mit den Begriffen ermergence (Entstehen/Aufkommen) und emergency (Notfall) umschrieben. Als AIDS Millionen von Opfern forderte, war die erste öffentliche Reaktion im Westen Unglaube, Erstaunen und schließlich Panik. Inzwischen ist die Welt mit einer Reihe von emerging diseases konfrontiert, wie AIDS, SARS oder Vogelgrippe üblicherweise genannt werden. In Kanada, China und anderswo waren die Tourismusindustrie und der Luftverkehr durch die Vogelgrippe eingeschränkt, und es wurden Tausende von Hühnern vernichtet, wenn in einem Land der H5N1Virus an einigen Brutstätten gefunden wurde. Alle drei Krankheiten (und sie sind nur die bekanntesten) sind infektiös, werden durch Viren übertragen und haben das Potenzial, schnell zu einer weltweiten Epidemie zu werden.

In allen drei Fällen reagierten die Gesundheitsbehörden ursprünglich wie bei einem Notfall mit Eindämmungsmaßnahmen für potenziell infizierte Menschen, Quarantäne und speziellen Verfahren der Untersuchung und Kontrolle. Viele dieser Maßnahmen hatten experimentellen Charakter, und es schien, als führte eine neue Qualität der Zusammenarbeit weltweit zu neuen Forschungsmustern und einem neuen Verständnis von Infektionskrankheiten.

\section{Die aktuelle Herausforderung für die Kontrolle von Infektions- krankheiten - eine Krise der strategischen Konzeption?}

Jährlich sind 15 Millionen Todesfälle auf Infektionskrankheiten zurückzuführen, was 26 Prozent der weltweiten Sterblichkeitsrate ausmacht. Vorhandene 
Medikamente und Impfstoffe hätten einen großen Teil dieser Tragödie verhindern können, zu der es in erster Linie in den armen Regionen der Erde kommt. Allein drei Krankheiten - AIDS, Tuberkolose (TB) und Malaria sind für 39 Prozent der Todesfälle durch Infektionskrankheiten verantwortlich, während durch den Zugang zu Lebensmitteln und sicherem Trinkwasser weitere 2 Millionen Todesfälle verhindert werden könnten.

Die Sterblichkeitszahlen lassen jedoch nur einen Teil der aufkommenden Katastrophe erkennen. Misst man die Gesundheitsbeeinträchtigung anhand von schwer wiegenden und dauerhaften Behinderungen, ist fast ein Sechstel der Weltbevölkerung betroffen - also 1 Milliarde Menschen. Zu diesen Behinderungen gehören insbesondere Beeinträchtigungen beim Heranwachsen und bei der geistigen Entwicklung von Kindern auf Grund von Schistosomiasis, geistige Defizite bei Überlebenden von Meningitis und Erblindung durch Onchozerkose. Von diesen „versteckten“ Krankheiten sind fast ausschließlich extrem arme Menschen betroffen, die in entlegenen Gebieten außerhalb der Reichweite von gut zugänglichen und regulären Gesundheitsdiensten leben. Da die Sterblichkeit bei diesen Krankheiten relativ gering ist, wurde ihnen trotz hoher Erkrankungsziffer und der damit verbundenen hohen sozialen und wirtschaftlichen Kosten in der Vergangenheit von Regierungen, Nichtregierungsorganisationen und der internationalen Gemeinschaft vielfach nur geringe Bedeutung beigemessen.

Andere Infektionen flackern auf wie Spitzen oder Plateaus eines Elektrokardiogramms, wenn man ihr Auftreten über der Zeitachse zeichnet. Hierbei handelt es sich um emerging diseases, die den Hang zur Epidemie haben. Sie haben verheerende Folgen, machen über Monate in vorhersagbaren Regionen oder Jahreszeiten Schlagzeilen und kommen für Fachleute des Gesundheitswesens anfänglich oft völlig überraschend. Ebola, AIDS, SARS und Vogelgrippe beherrschen seit ihrem ersten Auftreten die Schlagzeilen.

Einige Krankheiten wie Malaria und Cholera sind erneut aufgetreten (reemerged) und zeigen uns damit, dass infektiöse Krankheitserreger im Vergleich zu früheren Mustern eine sich ständig wandelnde Bedrohung darstellen können. Mikroben wie auch Übertrager nutzen jede Gelegenheit, sich zu vermehren, zu mutieren, zu migrieren, sich an andere Wirte und Lebensräume anzupassen und sich so weiter zu entwickeln, dass sie gegen Medikamente 
und Insektizide resistent werden. Ein solches Verhalten ist nicht „intelligent“ oder „klug“; es folgt lediglich den Darwin’schen Gesetzen der Artenentwicklung und des „Überlebens des Passendsten“.

\section{Sorge, Engagement und neue Partnerschaften}

Nach dem Aufkommen von HIV/AIDS in den achtziger Jahren, als noch die weit verbreitete, aber falsche Auffassung vorherrschte, dass starke Antibiotika und Insektizide der Ära der Infektionskrankheit automatisch ein Ende bereiten würden, hat ein neues Nachdenken und eine neue Einschätzung der aktuellen globalen Krankheitssituation zu völlig neuen Ansätzen und Konzepten bei der weltweiten Eindämmung von Infektionskrankheiten geführt. Szenarien einer neuen Zeitperiode des „schwarzen Todes“ gelangten in die Denkfabriken der Verteidigungsindustrie und beflügelten die Vorstellung einer kleinen Anzahl von „Bioterroristen“ - hielten aber auch Einzug in die Hollywood-Studios. Die globalen Szenarien legten besonderen Nachdruck darauf, dass der Vorsprung von Wissenschaft und Medizin gegenüber den Kräften der Natur verloren gehen würde. Die Untergangsstimmung dieser Periode in den frühen neunziger Jahren führte in der wissenschaftlichen Gemeinschaft dazu, dass die Forschung von einer maximalen und oft unbürokratischen Zusammenarbeit geprägt wurde. Begünstigt wurde diese Entwicklung durch eine zunehmende Geschwindigkeit der Informationsübertragung über Internet und Satelliten.

Die zunehmende Geschwindigkeit und das Volumen des internationalen Reiseverkehrs hatten den Ausbruch einer Epidemie in einem Teil der Welt zu einer potenziellen Bedrohung an jedem anderen Ort gemacht. Die Fähigkeit von Infektionskrankheiten, Gesellschaften und Volkswirtschaften zu destabilisieren, wurde von AIDS und SARS auf alarmierende Weise verdeutlicht und vermittelte die Botschaft, dass eine lokale Infektionskrankheit globale Konsequenzen für die Sicherheit haben kann.

Eine Resolution der Weltgesundheitsversammlung 2001 über „Global Health Security: epidemic alert and response“ verwies auf Anzeichen für eine Verbindung zwischen der Globalisierung des Handels und Reisens mit der jüngsten Zunahme von Infektionskrankheiten. 
Das Ende des Kalten Krieges hatte auch Konsequenzen für das allgemeine Sicherheitsempfinden. Während Sicherheit damals ausschließlich als eine Angelegenheit der territorialen militärischen Verteidigung angesehen wurde, haben die Kräfte der Globalisierung deutlich gemacht, dass in einer verflochtenen Welt zuvor weit entfernte Bedrohungen für die Gesundheit nun sofort und universell von Bedeutung sind. Die Auswirkungen von Gesundheitskatastrophen in irgendeinem Teil der Welt überschreiten mühelos Grenzen und durchdringen Gesundheits- und Sozialpolitik in einer Art und Weise, der man mit traditionellen Mitteln auf nationaler Ebene allein nicht mehr entgegenwirken kann.

Demzufolge entstand ein neuer mächtiger Trend: die systematische Entwicklung neuer Allianzen zwischen nationalen Gesundheitsbehörden, internationalen Organisationen, NGOs, Privatwirtschaft und akademischer Welt.

Gegen TB und Malaria geht man nun in breit angelegten Zusammenschlüssen von Partnern an mehreren Fronten vor. Das ursprüngliche Behandlungskonzept für TB - DOTS - wird mittlerweile in zahlreichen Ländern eingehalten. Es stellt eine ununterbrochene Versorgung mit hochwertigen Medikamenten sicher und macht Medikamente für TB-Erreger, die gegen mehrere Mittel resistent sind, zu einem Bruchteil ihrer normalen Kosten verfügbar. Neu wurden auch die lokal tätigen Ärzte ins Gesamtkonzept des Programms integriert; sie tragen dazu bei, dass Fälle schnell erkannt werden. Wenn TB in Verbindung mit HIV auftritt, wurden Anreize geschaffen, damit Hilfsgemeinschaften und integrierte Dienstleistungen benutzt werden.

Die neue Partnerschaft Roll Back Malaria hat sich bewährt, und sie ist darauf vorbereitet, mit massiven Diagnose- und Medikamentenaktivitäten, mit der Verteilung von imprägnierten Moskitonetzen und mit anderen Schutzmaßnahmen in vielen Ländern ihren Beitrag zu leisten.

Auf der Tagesordnung der Außenpolitiker, die sich für mehr internationale Sicherheit einsetzen, steht deshalb immer öfter auch die Problematik der Infektionskrankheiten als Sicherheitsbedrohung, mit der nicht nur im Hinblick auf Bioterrorismus umgegangen werden muss.

Unerwartete Ausbrüche von Krankheiten sind eine enorme Belastung für anfällige und unterfinanzierte Gesundheitsstrukturen und ein erheblicher 
Störfaktor für Routinekontrollprogramme und Gesundheitsdienste. Die im Zusammenhang mit diesen Krankheiten entstehenden Belastungen halten oft über einen längeren Zeitraum an; sie verursachen enorme Kosten und beanspruchen menschliche und materielle Ressourcen, die für deren Eindämmung gebraucht werden. Die WHO-Resolution zur ,globalen Gesundheitssicherheit" hat formell die wichtigsten Ursachen für das Wiederkehren der Bedrohung durch Infektionskrankheiten und ihre Konsequenzen anerkannt, denen nur mit globalen Lösungen begegnet werden kann.

\section{Maßgebliche Ursachen der globalen Krise wurden oft übersehen}

Während die beschleunigte Globalisierung und mehr internationale Reisen Menschen und Krankheitserreger in engeren und häufigeren Kontakt gebracht und damit die Übertragung von Krankheiten begünstigt haben, stellt sich nun heraus, dass die Selbstgefälligkeit in Medizinerkreisen des öffentlichen Gesundheitswesens und in der Verwaltung ein ganz entscheidender Grund für den Zusammenbruch von Kontroll- und Überwachungsprogrammen war, die viele Krankheiten und Überträgerpopulationen in Schach gehalten hatten. Übermäßiges Vertrauen in die Macht von Insektiziden führte in den fünfziger Jahren zu der falschen Annahme, dass die Ausrottung von beispielsweise Malaria nur eine Frage der finanziellen Ressourcen und damit praktisch in greifbarer Nähe sei. Die Forschung im öffentlichen Gesundheitswesen trödelte bei der Entwicklung von Medikamenten und Impfstoffen. In Verbindung mit dem verbreiteten Mangel an operativen Einschätzungen, beispielsweise der Einhaltung von Einnahmeanweisungen durch Patienten und der Effizienz der gesundheitlichen Grundversorgung, haben das nachlassende Interesse an der Überträgerkontrolle in der postkolonialen Ära sowie die verbreitete Verwendung von insektiziden Substanzen in der Landwirtschaft zu einer erhöhten Resistenz der bedeutendsten krankheitsübertragenden Insekten gesorgt. Das Aufkommen „vernachlässigter“ tropischer Krankheiten hat eine enorm negative Auswirkung auf die Lebensmittelsicherheit, zerrüttet die traditionellen Formen der Landnutzung, behindert Bildung durch Absenzen in der Schule, verursacht Umsiedlungen von Bevölkerungsgruppen und sorgt dafür, dass künftige Generationen in ländlicher Armut verstrickt bleiben. Die Migration vom Land in die Städte führt zu schnellen und ungeplanten Urba- 
nisierungen mit den damit verbundenen Formen von Infektionsausbrüchen wie etwa von Stadtgelbfieber in Westafrika. Die Schlafkrankheit verursacht Schätzungen zufolge jährlich einen Verlust von 4,5 Mrd. US-Dollar allein in der Landwirtschaft.

Diese und andere Krankheiten sind nun in das internationale Blickfeld ehrgeiziger neuer Initiativen gerückt. Es scheint, als habe die frühere Selbstgefälligkeit ein Ende und als nähme man die Bedrohung durch Infektionskrankheiten in all ihren zahlreichen Dimensionen nun ernst.

Die neue Welle von öffentlich-privaten Partnerschaften stützt sich auf eine gemeinsame Realität: Die angegangenen Probleme sind so komplex, dass kein Beteiligter allein - sei es aus der Wirtschaft oder aus dem humanitären Bereich - hoffen kann, auch nur ein einziges Krankheitsproblem im Alleingang lösen zu können. Viele dieser Partnerschaften zielen darauf ab, Krankheiten auszumerzen, die die Menschheit seit Jahrhunderten plagen; andere bemühen sich an Universitäten und in der Industrie um die kostenintensive und riskante Erforschung und Entwicklung von Medikamenten und Impfstoffen, die keinen kommerziellen Markt haben, da von den Zielkrankheiten fast ausschließlich die Armen betroffen sind. Die fruchtbare Interdisziplinarität von so unterschiedlichen Fachgebieten wie Epidemiologie, Sozialforschung, Neurobiologie, Telekommunikation, Stadtplanung, Verkehrswesen und Kulturgenetik hat in jüngster Zeit Quantensprünge gemacht, die eine Diagnose des Ausbruchs von Infektionskrankheiten in entlegenen Gebieten fast in Echtzeit möglich gemacht haben.

\section{Neue Informationssysteme im Gesundheitswesen}

Geografische Informationssysteme und Erdbeobachtung über Satelliten sind Produkte des Kalten Krieges, die von Verteidigungsministerien für militärische Zwecke entwickelt wurden. Mit ihren Fähigkeiten der Abbildung von z.B. Temperatur, Bodenarten, Vegetationsabdeckung, Mustern der Landnutzung, der präzisen Lokalisierung von Wasserkörpern und Bevölkerungszentren, Straßen und Infrastruktur hätten sie auch als leistungsfähige Hilfsmittel für die Überwachung und Kontrolle von Infektionskrankheiten entwickelt worden sein können. Insbesondere die vielen vernachlässigten Krank- 
heiten, die sich durch Überträger ausbreiten, hätten erfasst werden können. Die genannten Informationsmittel eignen sich gleichermaßen für die Untersuchung von Ausbrüchen und für die Planung der Reaktion, wobei die zügige Lokalisierung von Fällen, eine schnelle Kommunikation und schnelles Abbilden der Dynamik einer Epidemie von entscheidender Bedeutung sind.

Noch vor einigen Jahren machten die Kosten für Hardware und die Komplexität der Computer-Modellierung den routinemäßigen Einsatz dieser Tools für Untersuchungsteams vor Ort unmöglich. Doch die Preise für Computer-Hardware und -Software sind gefallen, und neue zivile Satelliten befinden sich nun über fast allen Ländern in der Umlaufbahn.

Die WHO hat ein benutzerfreundliches Tool entwickelt, das den Namen Healthmapper trägt. Es bietet Anwendungen für die Einschätzung des Ausbruchs einer einzelnen Krankheit oder für die integrierte Überwachung und Kontrolle mehrerer räumlich und zeitlich miteinander verflochtener Krankheiten.

Eine weitere Technologie des Weltraumzeitalters - das Global Positioning System GPS - kann mit einfachen und kostengünstigen Handheld-Geräten verwendet werden und ermöglicht die Nutzung dieser leistungsfähigen Technologien bei der Arbeit in abgelegenen Orten in den Entwicklungsländern.

Ende 2002 wurde der Global Atlas of Infectious Diseases eingeführt, der nun breite Anwendung bei der Überwachung und Kontrolle von Krankheiten findet. Über 300 Indikatoren für mehr als 20 Infektionskrankheiten, die eine besondere Gefahr für die Volksgesundheit darstellen, wurden erfasst. Das Zusammenfassen von

- demografischen Daten,

- sozioökonomischen Bedingungen,

- Umweltfaktoren und

- meteorologischen Trendinformationen

in einem elektronischen Datennetz ermöglicht die Analyse und Vorhersage einzelner Krankheitsausbrüche oder allgemeine Schätzungen der Übertragung verschiedener Krankheiten. Hierdurch wird ein integrierter Ansatz der 
Überwachung gefördert, die ein breites Spektrum an entscheidenden Faktoren mit Einfluss auf die gesundheitliche Lage in genau eingegrenzten Gebieten berücksichtigt.

Der Einsatz von Landkarten als klassische Form der Visualisierung und die Überlagerung mit unterschiedlichen Merkmalen wie

- Anzahl der Dörfer, die mit Schistosomiasis infiziert sind,

- Verfolgung der Polio-Überwachung in denselben Dörfern,

- Stand der Chloroquin-Resistenz in dem gesamten Gebiet,

- Stand der Programme von ITTNs im selben Gebiet,

- Geschichte von Masernfällen in denselben Dörfern und

- EPI-Impfstatus

verleihen der dezentralisierten Planung und Einschätzung einen kräftigen Impuls. Der globale Atlas bietet auch einen einzigen Zugangspunkt zu Daten, die von verschiedenen elektronischen Überwachungssystemen erfasst werden. Er ist für das Verfolgen von einzelnen Krankheiten eingerichtet, die sich durch eine sich schnell wandelnde Dynamik auszeichnen. Viele dieser einzelnen Datasets (FluNET, DengueNet usw.) können per Ferneingabe von Daten durch Forscher und Überwachungspersonal in anderen Ländern aktualisiert werden.

\section{Aufkommende Krankheiten - aufkommende Lösungen?}

Während Technologien in den letzten Jahren - zumindest theoretisch - einen Einfluss auf die Gesundheitsforschung und Seuchenbekämpfung hatten, ergibt sich ein weniger rosiges Bild, wenn man sich die Abdeckung ansieht, die einen vollständig lokalen Ansatz erfordert, den viele Länder und Organisationen noch nicht im Blick haben. Vielfach werden Gesundheitsprogramme von „hoher Qualität“ nämlich ausschließlich als professionelle Aufgabe mit einem klaren Top-down-Ansatz betrachtet, obschon man Lippenbekenntnisse zu partizipierender Forschung und Entwicklung abgibt.

Bei der Planung der kontinuierlichen Verbesserung der Effizienz bei der Eindämmung von Infektionskrankheiten muss man sich für Systeme interes- 
sieren, bei denen Einzelpersonen globale Situationen einschätzen, auf koordinierte und regelmäßige Weise zusammenarbeiten und ganz nüchterne Alltagsprobleme bei Zahlungen, Medikamentenlieferungen und Transporten lösen. Wenn das nicht der Fall gewesen wäre, wäre die medizinische Grundversorgung in ländlichen Regionen weitgehend zusammengebrochen.

Die lokal im Gesundheitswesen tätigen Menschen müssen mehr Eigenständigkeit in ihren Entscheidungen bekommen, damit sie mehr auf ihre Nachbarn achten, statt auf Befehle von oben zu warten.

Durch lokales Denken und Handeln legen ländliche Bevölkerungen globales Verhalten an den Tag, indem sie kontinuierlich über die Jahreszeiten und Dürrezeiten hinweg die verfügbare Energie und Zeit für prioritäre Maßnahmen in der Landwirtschaft, Gesundheit und Bildung aufwenden. Kein Einzelner kann diese Variablen allein einschätzen. Seine Wahrnehmung aus dem Blickwinkel der Straße ermöglicht keine „Vogelperspektive“, aus der sich das gesamte Gesundheitssystem überblicken lässt, und er weiß nicht, wie er die begrifflichen Hilfsmittel eines kognitiven Apparats einsetzen soll, die einer solchen Ansicht erst ihre Aussagekraft verleihen würden.

Das lokale Feedback, das all diesen Systemen innewohnt, kann sich durchaus als das Geheimnis für dezentralisierte Planung entpuppen, denn eine im Gesundheitswesen tätige Person (Community Health Worker, CHW) weiß zu keinem Zeitpunkt, wie viele andere CHWs im ganzen Land gleichzeitig an demselben Problem arbeiten.

Wenn wir versuchen, ein System mit landesweiter Abdeckung aufzubauen, das für das Lernen von Grund auf konzipiert ist, ein System, in dem Makrointelligenz und Anpassungsfähigkeit aus lokalem Wissen abgeleitet werden, gilt es, eine Reihe von Grundsätzen zu berücksichtigen:

- More is different. Dieser Slogan der Komplexitätstheorie erfordert in unserem Fall, dass eine kritische Masse von CHWs eine intelligente Datenerfassung durchführen muss, um eine globale Einschätzung zu ermöglichen. Aber „More is different" bezieht sich auch auf die Unterscheidung von Mikromotiven und Makroverhalten. Freiwillige Mitarbeiter auf Gemeindeebene, die Daten sammeln, „wissen“ nicht, dass sie Prioritäten 
für Wege von strategischen Gesundheitsentscheidungen auf Landesebene vorgeben.

- Unkenntnis ist hilfreich. Die Einfachheit bei der Erkennung von Anzeichen und Symptomen bei der Überwachung ist ein Leistungsmerkmal und kein Bug (nach der Meinung von Software-Entwicklern). Emergente Systeme werden schwerfällig und schwer zu handhaben, wenn sie „überladen“ sind, oder ihre Bestandteile werden übermäßig kompliziert. Es ist besser, ein dicht verwobenes System mit einfachen Elementen zu bauen und das komplexere Verhalten von selbst entstehen zu lassen. Über einzelne CHWs zu verfügen, die in der Lage sind, den Zustand des gesamten Systems einzuschätzen, kann für die „Schwarm-Logik“ (das ist das, was man macht, wenn man lokal organisierte Grundüberwachungs- und Kontrollsysteme aufbaut) ein echter Nachteil sein (aus demselben Grund will man nicht, dass eines der Neuronen im Gehirn plötzlich „empfindungsfähig“" wird!).

- Fördern von zufälligen Begegnungen. Dezentralisierte Gesundheitssysteme hängen in hohem Maße von zufälligen Interaktionen ohne zuvor festgelegte Anweisungen ab. Solche Treffen in ländlichen Gebieten und zwischen Dörfern und Personen sind für sich genommen willkürlich, doch weil so viele Personen im System sind, ermöglichen die Treffen den Menschen letztendlich, den Makrozustand ihres Systems selbst zu beurteilen und zu ändern. Ohne solche zufällige Treffen wäre ein humanes Ökosystem nicht in der Lage, neue Lösungen zu finden oder sich ständig an neue Umweltsituationen anzupassen.

- Mustererkennung durch Zeichen. Trotz eines begrenzten wissenschaftlichen Vokabulars verlassen sich Dorfbewohner auf Muster in den Zeichen und Symptomen, von denen sie wissen, dass sie auf Krankheiten hindeuten, die zuvor schon einmal aufgetreten sind. Änderungen von Mustern lösen erhöhte Wachsamkeit aus und ermöglichen das Kursieren von Metainformation durch Siedlungen und ganze Gebiete.

- Achte auf deine Nachbarn. Dies ist wahrscheinlich die wichtigste Lektion, die wir aus der ländlichen Wahrnehmung von Gesundheit lernen können, die gleichzeitig die weit reichendsten Konsequenzen hat. Der primäre Mechanismus von „Schwarm-Logik“ ist die Interaktion von Personen vor Ort, die in zufälligen Treffen übereinander stolpern. Hohe Mobilität innerhalb eines bestimmten ländlichen Gebietes wird mehr Interaktion zwischen Nachbarn und Reisenden erzeugen und letztendlich 
Lösungen für Probleme hervorbringen und langfristig für Selbstregulierung sorgen.

Beim Konzipieren eines Lösungsansatzes für die Einbeziehung von Rotem Kreuz und Rotem Halbmond in Ausbrüche von Infektionskrankheiten durch dezentralisierte Freiwilligennetzwerke und Niederlassungssysteme sollten drei Hauptkomponenten berücksichtigt werden:

- Der neue Modus des gemeinsamen Handelns von Beteiligten, der durch die wahrgenommenen Krankheitsbedrohungen und operativen Schwierigkeiten in den neunziger Jahren aufgekommen ist. Diese Systeme, ihre internen Regelungen für gemeinsames Handeln und „offene“ Kommunikation usw. geben wahrscheinlich die Richtung vor, die in Zukunft eingeschlagen werden muss.

- Die zurzeit verfügbare Telekommunikationstechnologie, verfügbare Datenbanken und Programmiermöglichkeiten, die weiter wachsen und von der WHO und anderen Organisationen getestet werden, bilden eine Grundvoraussetzung für angemessenes und strategisches Handeln im Roten Kreuz bei der Gesundheitsförderung auf Niederlassungsebene.

- Eine Überprüfung der Programme des Roten Kreuzes für Gesundheitsarbeiter in den Dörfern muss die Grundlage für einen realistischen und angemessenen Ansatz schaffen, bevor weit reichende technische Entscheidungen getroffen werden. Die aktuelle weltweite Wahrnehmung von Emergenz in der Systemanalyse sollte eine entscheidende Rolle bei dieser strategischen Prüfung spielen. 